\title{
AGRICULTURE, MARCHÉ ET EXTRAVERSION DANS LES MONTAGNES D'AFRIQUE DE L'EST
}

\author{
AGRICULTURE, MARKET AND ECONOMIC \\ EXTRAVERSION IN EAST AFRICAN MOUNTAINS
}

\author{
Sylvain Racaud*
}

\begin{abstract}
Résumé
Cet article interroge des mécanismes de reproduction d'inégalités entre des acteurs et entre des territoires à partir de l'analyse d'une économie rurale africaine basée sur le vivrier commercialisé. Il indique que les modalités d'intégration de l'agriculture montagnarde au marché maintiennent une économie rurale extravertie, c'est-à-dire subordonnée à l'extérieur, entretenant des relations dissymétriques qui freinent l'accumulation et le développement dans la campagne. En s'appuyant sur une rente, l'exemple des montagnes Uporoto au sud-ouest de la Tanzanie illustre des mécanismes de sous-développement. Les flux de produits non transformés sont émis vers des marchés extérieurs sans valorisation locale et la rente est captée par des acteurs à l'interface de l'urbain et du rural. Le propos se structure en deux temps : l'essor du modèle agricole intégré à l'urbain, puis les incertitudes de l'accès à un marché défaillant.
\end{abstract}

Mots-clés : Agriculture - montagne — intégration au marché - extraversion — économie de rente - Tanzanie

\begin{abstract}
From a cash food crop based rural economy analysis, this article is about reproduction's mechanisms of inequalities between actors and territories. It shows the integration of the mountainous agriculture to the market maintaining a rent-based economy. This economy has dissymmetric relations with the global market and its development is hindered. The Uporoto Mountains (South-West of Tanzania) is an example of mechanisms of underdevelopment. The flows of raw products are produced towards external markets without local increase in value. Actors located at the interface between urban and rural benefit from this rent-based economy. The article is structured in two parts: development of the agricultural model integrated to the urban areas, and uncertainties of access to the market.
\end{abstract}

Keywords: Agriculture - mountain - integration to the market - rent-based economy - Tanzania

Comment les modalités d'intégration de l'agriculture montagnarde aux marchés maintiennentelles une économie rurale extravertie ? En quoi l'orientation économique d'une montagne agricole périphérique illustre-t-elle des mécanismes plus globaux d'extraversion économique ? Bien que les transformations internes soient profondes, les économies africaines sont toujours marquées par un

* Géographe, Université Bordeaux-Montaigne, UMR 5115 Les Afriques dans le Monde 
modèle extraverti qui repose sur l'économie de plantation ou minière [Hugon, 2009], et la perpétuation de l'extraversion est une modalité majeure de relation de l'Afrique au monde [Magrin, 2013, p. 22]. Les orientations vers l'extérieur s'inscrivent dans le temps long, le contrôle des échanges a été un des moteurs principaux d'empires précoloniaux, en particulier ceux situés à des espaces d'interfaces [Iliffe, 2009]. Ensuite, le modèle colonial a mis en place les grandes cultures d'exportation sur lesquelles les États indépendants créés dans les années 1960 se sont appuyés. Les rentes agricoles demeurent toujours un pivot de l'économie de nombreux États du continent ${ }^{1}$.

Les montagnes d'Afrique de l'Est sont emblématiques de ces logiques d'extraversion et rentières. Ces régions sont des «terres d'élection de l'agriculture commerciale, elle-même motrice des économies coloniale et nationale » [Charlery de la Masselière et al., 2009, p. 311]. Leurs conditions environnementales offrent ainsi un large choix de potentialités et sont favorables à une agriculture variée, «la montagne introduit des gradients multiples et vigoureux en termes de température, de précipitations, de couverture végétale, de gamme de cultures, de ressources pastorales » [Bart, 2001, p. 9]. Les massifs concentrent une mosaïque de paysages et une diversité de ressources dont l'abondance a été un facteur d'attractivité pour les hommes. Par conséquent, les densités rurales sont fortes, souvent supérieures à $300 \mathrm{hab} . / \mathrm{km}$. Le fait montagnard, et cela d'autant plus en Afrique intertropicale, ne repose pas uniquement sur des particularités naturelles, il est un élément d'enjeux de développement de territoires spécifiques marqués par de fortes densités humaines.

Ces caractéristiques confèrent aux montagnes d'Afrique orientale des avantages comparatifs pour la production agricole et les échanges commerciaux dont l'exploitation a bouleversé les sociétés montagnardes et leurs rapports aux territoires. Les dynamiques en œuvre dans les territoires de montagne traduisent l'amplification des relations entre les villes et certaines campagnes, en particulier celles dans lesquelles ont été réalisées des reconversions agricoles. Le développement du vivrier marchand [Chaléard, 1996] est une réponse à la croissance de la demande urbaine et une alternative au tarissement des revenus issus d'anciennes filières exportatrices. Ce cadre de production et d'échange est régulé par le secteur privé et l'investissement de nouveaux acteurs intensifie les logiques marchandes dans l'organisation des espaces de montagne. L'objet de l'article est de montrer comment l'intégration de l'agriculture des montagnes Uporoto au marché contribue à la persistance d'une économie rurale extravertie, c'est-à-dire subordonnée à un environnement extérieur, établissant ainsi un lien de dépendance et une relation dissymétrique.

Les axes du recueil de données ${ }^{2}$ se rapportent aux caractéristiques socio-économiques des systèmes de production et à leurs évolutions, aux modalités d'accès au marché physique, à l'information et au crédit, aux modalités de transaction et au flux agricoles. Les principaux lieux d'enquêtes sont les marchés ruraux et dans une moindre mesure, des marchés urbains à Mbeya, la métropole régionale du sud-ouest tanzanien et également dans les trois principaux marchés de redistribution de vivres à Dar es-Salaam, c'est-à-dire sur une route marchande dont l'origine est une montagne agricole périphérique ${ }^{3}$.

Le propos est organisé en deux parties. La première porte sur l'établissement du secteur agricole fondé sur les cultures vivrières marchandes. La seconde met en avant les défaillances dans les échanges en traitant des incertitudes quant à l'accès aux marchés.

\footnotetext{
1. Une économie de rente est définie comme une «économie pouvant se reproduire sans accumulation grâce à l'exploitation de ressources naturelles ou de transferts financiers » [Hugon, 2012, p. 123].

2. Le matériau empirique est issu de questionnaires (258) et d'entretiens ( 74 formels et autant d'informels) réalisés avec des acteurs de la production et de la commercialisation des produits agricoles ainsi qu'avec des acteurs institutionnels.

3. Ce travail est issu d'une thèse de géographie au cours de laquelle quatre missions de terrain ont été effectuées entre 2008 et 2012 ; missions financées par le programme européen CREATING, le programme CORUS « Montagnes et villes moyennes en Afrique de l'Est » et une bourse de terrain de l'école doctorale TESC, UT2. Ce travail a été complété par une mission réalisée fin 2014 dans le cadre du projet MONDIS-MEDAF, Labex SMS référence ANR-11-LABX-0066.
} 


\section{D'un modèle agricole à un autre : nouveaux flux et permanence de l'extraversion}

Si l'orientation vers l'extérieur des économies rurales est le scénario le plus ordinaire, cette tendance prend des formes particulières dans les massifs agricoles, qui sont des supports anciens de cultures commerciales coloniales, et pour certains, des lieux privilégiés de diffusion du capitalisme agricole et des territoires en reconversion. Ces éléments seront développés après que la problématique et la notion d'extraversion aient été précisées.

\section{Extraversion, sous-développement, la partie et le tout}

L'orientation vers l'extérieur doublée du lien de subordination de l'agriculture de montagne établit un lien de dépendance, une relation dissymétrique, voire inéquitable, si l'on voulait glisser dans un débat moral. Ce lien est au cour de notre conception de l'extraversion de l'économie rurale montagnarde.

L'extraversion est une notion utilisée le plus souvent pour caractériser des économies nationales. En ce sens, elle se réfère à des flux qui circulent à l'échelle internationale. Dans le Larousse, l'entrée « extraversion » renvoie à «extraverti » au sujet d'une économie dont la croissance s'appuie sur l'intensification des échanges internationaux. Un dictionnaire d'économie et des sciences sociales fait référence à une économie nationale qui doit financer ses importations par l'exportation d'une part très élevée de sa production; plus loin, on lit «l'économie est subordonnée à des économies étrangères »[Echaudemaison, 2013, p. 215]. Selon la théorie dépendantiste, les mutations de l'économie mondiale déterminent la place périphérique de l'Afrique dont l'économie est extravertie et l'extraversion apparaît alors comme le registre de production des inégalités [Bayart, 1999]. L'extraversion concerne principalement les pays en développement. Les manifestations de ce phénomène sont entre autres l'exportation vers des marchés plus solvables et la sortie de capitaux.

L'extraversion freine le développement de l'économie de départ. Même si des changements ont lieu dans les campagnes africaines, tout comme le continent connaît des transformations accélérées et à des rythmes inédits, il n'en demeure pas moins qu'une grande part du monde rural reste faiblement pourvue en infrastructures basiques (en matière d'électricité, d'eau, de transport, de santé, de services, etc.). Le monde rural, essentiellement agricole, ne rencontre que faiblement le développement, c'est-à-dire, le progrès, le changement " positif », l'élargissement des choix offerts aux individus, la possibilité de vivre longtemps et en bonne santé, si l'on s'appuie sur la définition normative du PNUD [Magrin, 2013].

De l'échelle mondiale, nous déplaçons le curseur à l'échelle plus régionale en interrogeant la situation périphérique des montagnes Uporoto et leur intégration au territoire national. Ainsi, l'entité d'analyse retenue est une économie rurale montagnarde qui exporte vers des marchés extérieurs, en l'occurrence les marchés urbains, principalement dans le cadre national, et plus précisemment, celui de la capitale économique nationale. Des productions (banane plantain, maïs, pomme de terre) sont aussi exportées dans des pays de la sous-région avec des systèmes de commercialisation similaires (standardisation, types de conditionnement, types de transport, types d'acteurs ${ }^{4}$ ). L'exemple des montagnes Uporoto illustre, à une plus grande échelle, des relations dissymétriques à plus petite échelle. Autrement dit, l'orientation économique d'une montagne agricole périphérique est un exemple de mécanismes plus globaux d'extraversion économique.

Selon une conception systémique, la partie est dans le tout, mais il est moins évident d'accepter que le tout se retrouve dans la partie. Des principes de la totalité se retrouvent dans les parties, il est donc possible d'enrichir la connaissance de l'un ou de l'autre par l'un et l'autre, ce qu'Edgar Morin nomme le principe « hologrammatique » [Morin, 1977]. Si la partie illustre le tout, elle ne le résume pas, elle l'éclaire tandis que le tout informe sur la partie. Dans ce travail, la partie est l'économie

4. Cela est différent pour les cultures anciennes d'exportation (thé, café) ou pour les cultures destinées aux marchés européens que l'on peut trouver dans d'autres montagnes ou hautes terres est-africaines (par exemple, les haricots verts du Kenya). Ces filières internationales répondent à des standards internationaux, dont les chaînes de valeurs sont différentes de celles des cultures destinées au marché est-africain. 
montagnarde et le tout est l'économie africaine ${ }^{5}$. Basé sur une rente agricole, le modèle de développement dans les montagnes Uporoto enraye le développement de l'économie montagnarde. Cette rente ne produit pas d'accumulation. Les économies rurales des massifs est-africains continuent d'expédier des productions sans valeur ajoutée locale. Cela est à l'image de "l'Afrique [qui] est demeurée une "économie de rente", où le processus d'accumulation n'a pas pu être réellement enclenché » [Hugon, 2009, p. 9]. La croissance africaine «demeure dépendante d'une conjoncture mondiale » [Ibid, p. 27] et la croissance économique montagnarde repose sur des marchés urbains. À l'échelle de l'Afrique subsaharienne, «le revenu par habitant n'a presque pas augmenté en cinq décennies » [Magrin, 2013, p. 21], tandis que le monde paysan ne récolte que quelques miettes de l'exploitation des ressources agricoles montagnardes. Structurellement, le marché continue de s'appuyer sur une faible rémunération des producteurs. Le commerce du vivrier marchand renforce le lien de subordination entre la société paysanne et les marchés urbains.

\section{Reconversion agricole et orientation vers le marché urbain}

Les différentes conditions de l'échange ont influencé la production qui s'opère de nos jours dans le cadre d'une urbanisation accélérée et d'un marché dominé par des acteurs privés. L'ouverture des montagnes d'Afrique de l'Est remonte à plus de dix siècles. Tandis que les circuits commerciaux d'esclaves connectaient l'Afrique orientale au golfe Persique et à l'Inde dès le IX $\mathrm{X}^{\mathrm{e}}$ siècle, à la même période, la banane originaire d'Asie a permis à des cultivateurs de coloniser les pentes du Kilimandjaro. Puis, des marchés permanents dédiés à l'échange des spécialisations régionales parsemaient les piémonts du toit de l'Afrique et du mont Kenya [Iliffe, 2009]. À partir du XX ${ }^{\mathrm{e}}$ siècle, l'ouverture rurale montagnarde a essentiellement reposé sur la production et l'exportation de thé et de café. Les colons ont introduit le café, puis le thé, vers le dernier quart du XIX ${ }^{\mathrm{e}}$ siècle dans les montagnes des Grands Lacs. Au début du $\mathrm{XX}^{\mathrm{e}}$ siècle, les cultures d'exportation devinrent rémunératrices et elles se sont développées dans leurs étages de prédilection, soit entre 1300 et $1800 \mathrm{~m}$ pour la région. L'administration coloniale encouragea ces productions qui modifièrent profondément les rapports de production et les rapports sociospatiaux en assignant des populations à un territoire de production [Charlery de la Masselière, Racaud, 2012]. Ensuite à partir des Indépendances, les nouvelles administrations continuèrent à inciter l'agriculture de rente et encadrèrent les filières. Les cultures commerciales d'exportation ont engagé les paysans montagnards dans l'économie de marché et ont ainsi favorisé les mutations sociales et économiques.

En réponse aux faillites des États, les mesures de libéralisation économique imposées par les bailleurs de fonds visaient à créer un marché incitatif et donc à terme, une économie compétitive. Globalement, les performances de l'agriculture ont diminué dans les années 1980, tandis que la part des produits alimentaires importés augmentait. Dans les années 1990 et 2000, les cours des matières premières ont beaucoup fluctué et par leur imprévisibilité, ils ont affecté non seulement les populations rurales, mais également les classes moyennes urbaines. Le processus progressif et continu de libéralisation a brisé les monopoles d'État et a dérégulé le commerce et les prix. Les structures d'encadrement de la production et de la commercialisation furent démantelées et les marchés n'étaient plus garantis. Les paysans se trouvèrent livrés à eux-mêmes dans un cadre concurrentiel inédit. Ces changements de l'environnement économique et en particulier ce contexte d'incertitudes se sont traduits dans le rural par des stratégies d'adaptation au jeu de l'offre et de la demande, puisque l'approvisionnement en argent par les cultures commerciales anciennes devenait aléatoire. Les agriculteurs ont alors modifié leurs systèmes de production au niveau de la composition des cultures, de l'usage des intrants, des techniques, de la main-d'œuvre, de la gestion du sol et des modes de commercialisation. Ils ont privilégié les denrées à destination des marchés urbains croissants (principalement : maraîchage, banane, pomme de terre). Le vivrier marchand devint une source de revenus pour les ménages ancrés dans l'économie monétaire.

5. Cette notion d'« économie africaine » n'est pas une réduction excessive et encore moins une caricature. Elle garde une valeur heuristique (par exemple si l'on s'en réfère à des ouvrages tel L'économie de l'Afrique [Hugon, 2009] ou encore les travaux de J.-F. Bayart) et n'épuise pas la diversité des 54 pays africains si l'on accepte de penser la multiplicité dans l'unité [Morin, 1977]. 


\section{Économie agricole et amplification des flux dans les montagnes Uporoto}

Les montagnes Uporoto situées à la périphérie sud-ouest de la Tanzanie sont une des principales régions de production agricole qui pourvoient en vivres le marché national et en particulier celui de la capitale Dar es-Salaam [Racaud, 2013]. La population des Uporoto est d'environ un million d'habitants. Mbeya est la ville principale, elle compte près de 400000 habitants en 2012 [URT, 2013]. Elle cumule les fonctions administratives inhérentes à son statut de chef-lieu de district et de région et à celui de city $^{6}$. Cette métropole régionale est la porte d'entrée pour les pays voisins enclavés (Zambie, Malawi, sud-est de la République Démocratique du Congo) et elle est traversée par le corridor Dar es-Salaam - Zambie (train « Tazara » et autoroute du Sud « Tanzam »). Cette situation spécifique lui confère une position stratégique à l'échelle de la sous-région. La seconde ville est Tukuyu dont la population est d'environ 40000 habitants. Elle est le chef-lieu du district Rungwe. Hormis les habitants de Mbeya et de sa périphérie, ainsi que ceux de Tukuyu, la population est rurale.

Carte 1 - Situation des montagnes Uporoto

6. Un district est l'équivalent d'un département français. Mbeya est le chef-lieu du district Mbeya Rural et de la région Mbeya qui compte huit districts. Les districts Mbeya Rural, Rungwe et Mbeya city sont compris dans les montagnes Uporoto. 


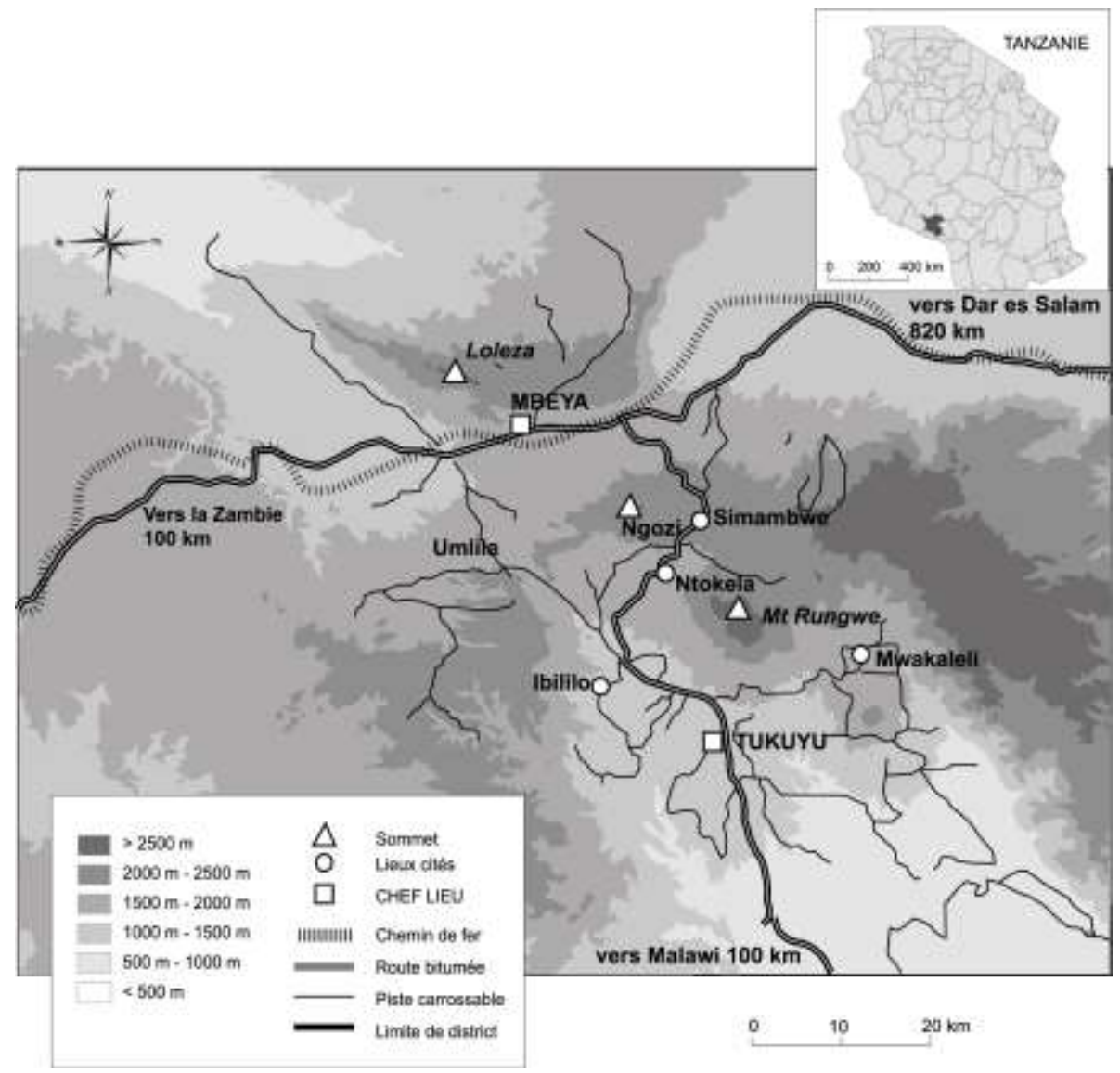

Source : Sylvain Racaud, réalisation V Alfaurt

L'économie urbaine et l'économie rurale sont considérablement imbriquées et dépendent du commerce des produits de la terre. Le secteur agricole anime l'essentiel de la population rurale et près de la moitié des habitants de la métropole. Le recensement de 2002 indique que $44 \%$ de la population de la ville de Mbeya, $80 \%$ de la population dans le district de Mbeya Rural et $90 \%$ de la population dans le district du Rungwe tirent leurs revenus de l'activité agricole [URT, 2011]. Cependant, les entretiens menés avec plusieurs agents de l'administration territoriale de la capitale régionale révèlent que ce seraient les trois quarts des urbains qui dépendraient de la production agricole ou de son commerce comme source de revenus.

L'agriculture est essentiellement familiale, les unités de production sont de tailles réduites. Elles comptent en moyenne cinq personnes et les superficies cultivées par foyer sont faibles, elles sont en moyenne de 0,9 hectare $^{7}$ [Racaud, 2013]. Cela est à mettre en perspective avec les fortes densités qui dépassent les 150 habitants par $\mathrm{km}^{2}$ et qui peuvent atteindre 400 habitants par km² sur les versants sud du mont Rungwe, au sud de l'arrête des Uporoto. Les foyers cultivent entre trois et quatre parcelles de petites dimensions et la main-d'œuvre est principalement familiale. Cependant, près de

7 L'unité localement utilisée est l'acre anglo-saxonne, une acre représente 0,4 ha, c'est-à-dire $4000 \mathrm{~m}^{2}$ environ ou un carré d'à peu près 63 mètres sur 63 mètres, 0,9 hectare équivaut à 2,3 acres. 
la moitié des exploitations ont recours aux journaliers à des moments précis du calendrier agricole pour des tâches spécifiques, par exemple pour la récolte des pommes de terre qui est une culture intensive. Dans les années 1980, l'essor de cette dernière comme production commerciale a fortement contribué à la pénétration de l'économie monétaire, à des phénomènes d'individuation et par conséquent au délitement des solidarités villageoises [Sokoni, $2001 ; 2008$ ]. La production fait appel à des techniques simples, par exemple le travail du sol est réalisé à la force des bras avec la houe, outil aratoire quasi exclusif utilisé pour le labour, le sarclage et la préparation des billons ; la traction animale est résiduelle. L'aménagement en terrasses est la technique culturale traditionnelle des hautes terres des montagnes Uporoto, il permettait de limiter l'érosion des sols. Leur entretien collectif, ancré dans le système coutumier de gestion des activités agricoles est tombé en déclin à cause de la commercialisation des moyens de production en réponse aux changements économiques [Sokoni, 2001]. La gamme des cultures s'est aussi adaptée aux évolutions du marché.

Le démantèlement de filières régulées par l'État et la croissance urbaine ont favorisé des changements dans les choix des productions. Les producteurs ont privilégié les denrées à destination de la demande urbaine grandissante, au détriment d'anciennes cultures d'exportation. Le pyrèthre a été remplacé par la pomme de terre et le chou dans une moindre mesure. Les caféiers du mont Rungwe ont été négligés au profit des bananiers. Actuellement, les principales cultures sont le maïs, la banane, la pomme de terre et le haricot. Leurs superficies et leurs tonnages augmentent sur la période 2002-2010. Le maïs est utilisé pour préparer le mets mangé quasi quotidiennement : l'ugali ${ }^{8}$. Cette céréale est essentiellement une culture vivrière, mais les surplus peuvent être vendus. La production de haricot croît fortement alors que les superficies augmentent peu, l'intensification traduit l'orientation marchande de cette légumineuse. L'essor de la banane plantain répond au développement du marché urbain. Son tonnage aurait crû de $58 \%{ }^{9}$ entre 2006 et 2010 . Cette plante est la principale production en volume des Montagnes Uporoto, elle est suivie par la pomme de terre. Ce tubercule est une culture alimentaire commerciale intensive dont le tonnage a aussi explosé, il aurait augmenté de $42 \%$ sur la même période. Les producteurs ont ainsi adapté leurs systèmes de production en plantant des cultures rapides et intensives pour générer des revenus rapides [Ponte, 1998].

L'intégration au marché de l'agriculture des montagnes Uporoto met en relation une plus grande variété d'espaces et d'acteurs. La carte suivante présente les directions principales des flux de produits émis depuis les montagnes Uporoto. L'orientation indique des relations avec le territoire national, principalement Dar es-Salaam et également avec les pays de la sous-région, montrant la situation centrale des montagnes Uporoto.

Carte 2 - Directions des flux agricoles depuis les montagnes Uporoto

8. L'ugali est une pâte réalisée à partir de farine de maïs bouillie et présentée sous forme d'une boule.

9. Calculs réalisés d'après des données récoltées auprès du Bureau régional de l'agriculture qui compile les données des districts. 


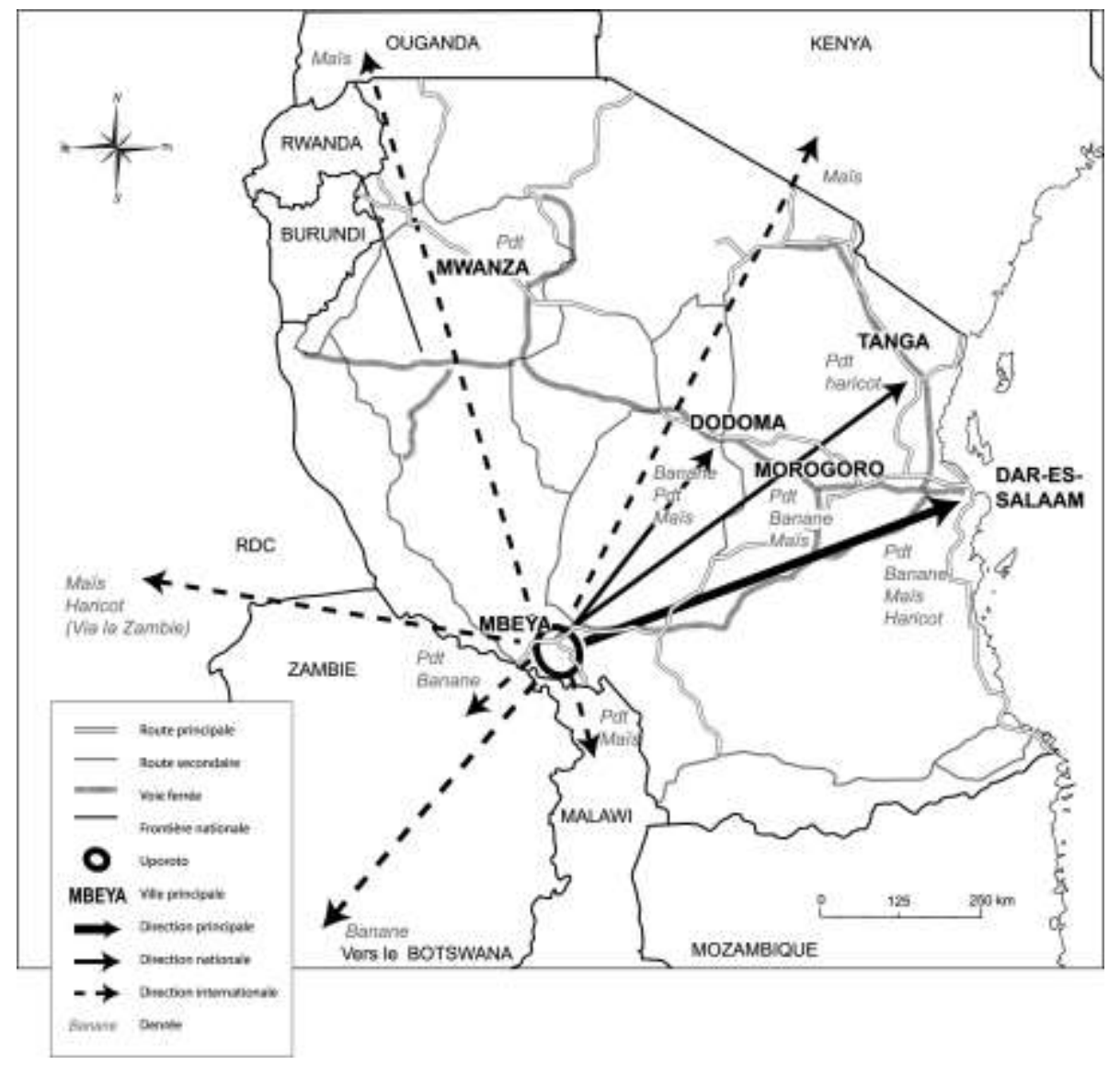

Source : Sylvain Racaud, réalisation V Alfaurt

L'État ne participe pas au marché du vivrier marchand comme il avait pu le faire par le passé pour le café, le thé et le pyrèthre en subventionnant les engrais et en garantissant les prix. L'essor de ce modèle agricole se réalise dans le cadre d'échanges régulés par le marché. De nouveaux acteurs ont investi les filières à tous les stades. Les filières s'allongent et se complexifient par l'intervention d'une gamme plus variée d'acteurs. Agentscourtiers, transporteurs, grossistes, détaillants, tout un éventail d'intermédiaires prend en charge les activités de mise en marché. Ils sont de différentes origines géographiques, par exemple, certains sont des ruraux locaux bons connaisseurs des terroirs, d'autres viennent de Mbeya, de Dar es-Salaam ou d'autres régions. L'attractivité exercée par ce secteur s'inscrit dans la continuité de celle qui avait motivé des migrants engagés dans la culture de la pomme de terre dès les années 1980. Certains acteurs impliqués dans le commerce agricole occupent un emploi principal dans la fonction publique en ville. Ces urbains ont un capital financier et éducatif, voire des positions stratégiques dans des organismes clés. Par exemple, M. «A », un responsable de l'agence gouvernementale des transports a investi dans l'acheminement de vivres entre Mbeya et Dar es-Salaam. Il possède aujourd'hui deux camions d'une trentaine de tonnes qu'il a fait venir de Belgique. Les acteurs économiques tels les commerçants, les banquiers, ceux du développement agricole et ceux de l'aménagement du territoire s'accordent pour dire que la région vit un «boom agricole». Le produit intérieur brut régional a augmenté de $19 \%$ entre 2006 et 2007 
et de $18 \%$ entre 2007 et $2008^{10}$ [URT, 2011]. Ce dynamisme est porté par des facteurs environnementaux, sociaux et historiques favorables et par l'augmentation de la demande, autrement dit par des éléments internes et externes à la montagne.

L'orientation agricole est tournée vers les marchés urbains et se traduit par une amplification des échanges. Dès lors, l'accès au marché est primordial.

\section{Incertitudes de l'accès à un marché défaillant}

L'ouverture de la montagne n'est pas nouvelle, ce qui est récent, ce sont les modalités d'articulation des paysans au système économique dérégulé. De plus, des accords bilatéraux, en particulier avec la Chine, partenaire commercial privilégié de la Tanzanie, encouragent l'assouplissement dans l'encadrement normatif des échanges. En matière d'échange de produits agricoles, la norme conquérante apparaît bien être la norme marchande dans un environnement global auquel les paysanneries sont soumises [Chaléard, 1999].

\section{Les lacunes de l'accès au marché physique}

Les montagnes Uporoto, comme généralement le monde rural africain, sont caractérisées par la faiblesse des infrastructures de transport et de stockage. La configuration du réseau routier ne favorise pas la fluidité des flux ; la qualité médiocre des infrastructures de transport augmente les risques de pertes, en particulier pour les produits frais rapidement périssables. Ces caractéristiques structurelles compliquent l'accès au marché et peuvent être des freins à l'intégration à ce dernier.

Le manque d'accessibilité qui est une contrainte majeure pour la participation aux échanges contribue au maintien de prix bas et à l'entretien de positions défavorables pour les producteurs en matière de pouvoir de négociation. En effet, la demande en denrées est moindre là où il est compliqué d'aller, c'est-à-dire dans les zones reculées seulement accessibles par des pistes (caillouteuses ou boueuses suivant la saison). L'offre supérieure à la demande conduit mécaniquement à des prix bas. Il existe d'importantes différences de prix entre les zones proches du bitume et celles qui en sont éloignées. Par exemple à Ntokela, village traversé par la route bitumée, un sac d'une centaine de kilos de pommes de terre se négociait au mois de juillet 2011 entre 22000 et $23000 \mathrm{TSh}^{11}$, alors qu'un sac similaire coûtait entre 15000 et 18000 TSh à Mwakaleli. La distance qui sépare ce village de l'axe bitumé n'est que de $30 \mathrm{~km}$, mais en saison sèche, il faut plus de deux heures en pick-up ou en camion pour parcourir cette piste non revêtue avec par endroits de fortes pentes, qui en saison des pluies ne peuvent être gravies qu'au prix d'efforts démesurés. Les prix indiqués montrent également qu'à Ntokela, l'écart entre le prix le plus bas et celui le plus haut est seulement de 1000 TSh alors qu'il atteint 3000 TSh à Mwakaleli. Plus un village est inaccessible, plus les écarts de prix d'achat y sont importants et plus le rapport entre l'offre et la demande est défavorable à l'offre, en l'occurrence aux producteurs.

Près des trois quarts des producteurs rencontrés sur les marchés périodiques utilisent ce dispositif local, dit gulio, comme moyen exclusif d'accès aux acheteurs. Ces marchés permettent de faciliter la circulation des marchandises en polarisant de manière ponctuelle les acteurs et les produits. Ils sont de hauts lieux des transactions, des interfaces entre le local et le lointain. Les producteurs indiquent que l'accessibilité est le critère principal dans le choix du lieu de vente. L'abondance de la clientèle est le second critère cité au sujet du choix des marchés. La plupart des paysans vendent dans un lieu par défaut, c'est-à-dire le marché proche, car s'ils avaient à choisir, ils privilégieraient un marché où la clientèle est plus abondante. L'insuffisance de capitaux pour transporter les marchandises vers des marchés plus rémunérateurs, par exemple en ville, explique alors en partie le moindre éventail des choix en matière d'écoulement des denrées. Les producteurs s'organisent très rarement pour vendre

10. Ces chiffres «mirobolants » sont à nuancer puisque le taux annuel d'inflation en Tanzanie est de $7,7 \%$ pour la période 2006-2010, il atteint $12,7 \%$ en 2012. Source Worldbank, 2013 et BAFD, OCDE, PNUD, OCEA, 2013. www.africaneconomicoutlook.org

11. Le shilling tanzanien est abrégé « TSh ». 10000 TSh équivalent à environ 5 euros. 
dans des lieux plus rémunérateurs en partageant les frais liés à cet acheminement. Il existe un seuil à partir duquel le rapport coût et bénéfice de la vente dans un grand marché urbain est plus avantageux que la vente en bord de champ, mais bien souvent compte tenu du faible volume, ce seuil ne peut être atteint individuellement [Dijsktra, 1999] et les paysans ne s'organisent pas. Bien que le mouvement coopératif ait été fort jusqu'au milieu des années 1970, la collusion avec le politique et les scandales de corruption qui l'entachèrent sonnèrent le glas de ces organisations. Un sentiment généralisé de défiance persiste à l'égard des divers types de mouvements coopératifs, surtout quand ils sont initiés par le pouvoir politique.

\section{Fragmentation du marché}

En l'absence de régulation étatique, le secteur privé a pris en charge l'entremise entre la production et la consommation. Il en résulte un marché fragmenté et opaque.

Les chaînes qui configurent le réseau d'échange sont plus nombreuses que les types de productions agricoles commerciales, car pour une même culture, plusieurs chaînes opèrent. Le modèle agricole est caractérisé par la coexistence de chaînes au nombre restreint d'étapes et d'autres marquées par un nombre élevé de stades de commercialisation pour un même produit et parfois au sein du même foyer. Le circuit principal relie un cultivateur à un intermédiaire, puis ce dernier à un grossiste ou un agent de Mbeya, lui-même rattaché à un grossiste de Dar es-Salaam. Néanmoins, des intermédiaires peuvent s'immiscer à chaque étape, les enquêtes montrent que les denrées peuvent passer entre les mains de cinq intermédiaires différents avant de parvenir au consommateur final.

La fragmentation du marché n'implique pas une dilution du pouvoir. Au contraire, cette caractéristique augmente la concentration et la puissance des intermédiaires situés à Mbeya, c'est-àdire à la charnière entre les campagnes et les grands bassins de consommation. Les intermédiaires ou agents collecteurs sont des acteurs qui mettent en relation les territoires montagnards avec le marché. Ils opèrent le plus souvent pour le compte d'un donneur d'ordre. Leur rôle est fréquemment décrié, ils peuvent être accusés de spolier la paysannerie. Pour autant, en l'état actuel du cadre des échanges, ils permettent à une grande partie des paysans d'accéder au marché et de dégager des revenus. Par ailleurs, les intermédiaires sont aussi régulièrement en forte compétition, comme en basse saison lorsque le marché est tendu. Ces périodes sont plus profitables pour les producteurs. Cependant, les cultivateurs ne peuvent que rarement spéculer parce que les produits sont périssables et parce qu'ils vendent généralement leurs stocks aussitôt les récoltes effectuées afin de pourvoir aux besoins immédiats en numéraire.

Le réseau d'échange est caractérisé par l'instabilité de ses formes qui font écho aux incertitudes du marché. Les variations s'expliquent surtout par la saisonnalité culturale, par les stratégies plus ou moins contraintes, et par le jeu de l'offre et de la demande. À cela s'ajoute le caractère incertain de l'activité agricole dépendante du climat. Ces traits mettent en avant l'incertitude structurelle de ce marché, ainsi que sa fragmentation inégale et variable. En raison du pouvoir situé en ville, les campagnes montagnardes sont subordonnées à des ressorts extérieurs qui sont le signe de l'extraversion. Cependant, la ville ne doit pas être simplement perçue comme un prédateur de la campagne puisque c'est bien la ville qui est motrice du vivrier commercialisé à travers la demande urbaine. La sujétion de la campagne peut alors apparaître quelque peu ambiguë, puisque si les rapports de force sont dissymétriques, si l'on observe peu d'accumulation dans le rural, la paysannerie parvient dans son ensemble à assurer la reproduction de ses moyens de production. Mais serait-ce son seul horizon dans un cadre d'échange dominé par les seules valeurs marchandes et l'opacité ?

\section{L'opacité de l'accès à l'information et l'informalité des transactions}


L'opacité quant à l'accès à l'information sur les prix domine le marché du vivrier commercialisé dans les montagnes Uporoto ${ }^{12}$. Il en résulte de fortes dissymétries de pouvoir dont disposent les agents économiques. Les producteurs ont un faible pouvoir de détermination ou de manipulation du prix. Cette position de faiblesse vis-à-vis du prix les distingue comme des «preneurs de prix » selon la terminologie économique souvent reprise par des acteurs institutionnels ${ }^{13}$. Les lacunes de l'accès au marché limitent leurs possibilités de recherche de meilleurs prix auprès d'autres acheteurs. De l'autre côté, les "faiseurs de prix » sont les acheteurs, en l'occurrence les commerçants et les intermédiaires. Ils disposent d'un pouvoir bien supérieur en matière de fixation et du prix. Ces agents sont mieux connectés au marché grâce à leur position plus en aval dans la chaîne et à leur localisation non confinée dans la campagne. L'accès dissymétrique à l'information sur l'état du marché et sur les prix renforce le désavantage des producteurs par rapport aux commerçants. Un tiers des cultivateurs confessent obtenir l'information sur les prix auprès du commerçant qui vient s'approvisionner de manière directe sur le lieu d'exploitation. Dans ce cas, l'information est fournie directement pendant la transaction.

Sur les marchés périodiques, il n'y a pas de dispositif officiel de diffusion de l'information. La moitié des paysans enquêtés déclarent découvrir l'information sur les prix en arrivant sur le marché ; le nombre de camions présents étant un indicateur de l'état de la demande. Les téléphones portables sont des instruments redoutables pour obtenir des informations, mais en 2012, seulement $10 \%$ des producteurs rencontrés en possèdent un. Par ailleurs, encore faudrait-il que les campagnes soient largement couvertes par des réseaux téléphoniques et électriques. D'un autre côté, les commerçants sont rompus à l'usage du téléphone portable qui est leur principal instrument de travail. Lors d'entretiens menés avec des commerçants et des intermédiaires, certains enquêtés firent la démonstration en utilisant leur téléphone, de la disponibilité sur le champ de l'information sur les prix à Dar es-Salaam ou dans une zone de production. Le répertoire téléphonique est une ressource prisée et de forte valeur. L'immédiateté et l'orientation en amont et en aval des sources d'information tranchent fortement avec l'accès très limité à l'information qu'ont les producteurs. De plus, les négociations sur les prix se font dans le cadre de transactions individuelles. Vendeurs et acheteurs du fait de l'inégal capital informationnel ne discutent pas sur un pied d'égalité. De plus, le téléphone ne peut pas tout et ne remplace pas la route. Il est important d'avoir accès à l'information, mais encore faut-il avoir un accès au marché physique et que l'investissement en vaille la peine.

L'imbrication du paysan au marché à travers l'essor des contrats « capitalistes etmarché » entre producteurs et commerçants organise la relation paysan et marché «autour des seules valeurs d'échange de biens indifférenciés, tous interchangeables » [Minvielle, 1999, P. 114]. Ces contrats sont marqués par l'informalité, ils sont oraux et ils reposent sur la confiance et les connaissances interpersonnelles. Ces contrats sont des accords entre deux acteurs, par exemple un producteur et un commerçant, un intermédiaire et un grossiste dont l'objectif est d'assurer la fourniture des marchandises. Des contrats à terme se développent pour les cultures intensives, telle la pomme de terre ou le chou, comme cela est devenu la norme depuis les années 2000 à Simambwe, haut lieu de ces productions. Si les contrats à terme se diffusent et facilitent l'accès aux intrants et au marché, l'exploitant perd son indépendance [Chaléard, 1999]. Ces accords sont passés oralement entre des commerçants et des paysans. Les négociations sont menées avant les récoltes, parfois même avant les semences et concernent la totalité de la production. Dans le but de garantir l'approvisionnement, le commerçant fournit une avance en numéraire pour faciliter l'achat d'intrants. D'autres types d'accords sont établis à la saison du ramassage lorsque les acheteurs sont en compétition pour trouver les marchandises qui ont pu être commandées depuis Dar es-Salaam. Les commerçants/agents procurent aux paysans les sacs et l'argent requis pour financer le paiement de la main-d'œuvre nécessaire à la récolte. Le paiement n'est pas effectué en totalité lors du ramassage, le paysan reçoit son dû dès que l'agent aura été payé par son donneur d'ordre ou que le commerçant aura revendu la

12. Ce marché n'en a bien évidemment pas l'exclusivité, mais dans notre cas, les carences des infrastructures de transport et de communication, le manque d'organisation des producteurs, etc. renforcent cette caractéristique. On est bien loin de la transparence des marchés prônée par de grandes institutions internationales.

13. Les expressions «prices takers » et «prices makers » ont couramment été employées, lors d'entretiens par des responsables de diverses administrations chargés du commerce, de l'agriculture, ou encore par l'IFAD (International Fund for Agricultural Development), l'agence internationale relevant des Nations Unies, en charge du développement agricole. 
marchandise à un autre opérateur. Le crédit relève de la confiance entre les acteurs de l'échange, mais il révèle aussi la position de faiblesse des producteurs, car le système d'échange n'offre pas toujours d'alternatives : l'offre peut être à prendre ou à laisser, sans garantie aucune d'en rencontrer une autre plus favorable. Les paysans des montagne Uporoto comme nombre de leurs homologues africains sont dans une position de dépendance. En effet, dès qu'ils s'accordent sur un contrat avec avance, les cultivateurs ne disposent plus d'autres options, ils ne peuvent plus profiter d'éventuelles meilleures opportunités. Le débouché étant limité à un acteur, le pouvoir de négociation est par conséquent très faible. En contrepartie, l'écoulement est assuré, tout comme les revenus, aussi moindres soient-ils. À ce moment-là, le paysan ne peut s'accorder sur un prix inférieur à ses coûts de production. Si ces types de contrats peuvent limiter les bénéfices engendrés, ils peuvent aussi réduire les pertes. Par exemple, le coût de production d'une acre de chou à Simambwe était de 200000 TSh (environ. $100 €$ ) et sa production s'est négociée entre 100000 et 600000 TSh lors des saisons précédentes, en fonction de l'état de l'offre et de la demande au plan national. Par ailleurs, les fluctuations de prix existent aussi dans des intervalles de temps très réduits, car les prix peuvent varier fortement au cours de la même saison. Compte tenu de la volatilité des prix, avec ces types de contrats, si les revenus sont limités, au moins il n'y a pas de pertes. Pour autant dans ce modèle agroéconomique incertain, ces types de contrats sont un choix par défaut, faute de mieux. Le producteur limite certes le risque, mais il hypothèque également des chances de revenus supérieurs.

En outre, des transactions sont réalisées à distance sans que les acteurs soient physiquement réunis. La dématérialisation des échanges concerne aussi le paiement qui peut être effectué par un transfert d'argent par le téléphone portable. Le système M-Pesa est un dispositif qui utilise les téléphones mobiles pour envoyer et déposer de l'argent. Ce procédé est très populaire, il a été lancé en 2007 au Kenya et un an plus tard en Tanzanie dans lequel il s'est très fortement développé. L'argent déposé sur un compte mobile peut être retiré auprès des innombrables petits comptoirs d'opérateurs téléphoniques. Ce fonctionnement est adapté aux lacunes du secteur bancaire classique, il représente une alternative pratique pour faciliter les flux d'argent sans qu'il y ait besoin d'un guichet bancaire. De surcroît, le manque d'accès au crédit pour les producteurs et le manque de standardisation des marchandises sont également symptomatiques d'un marché défaillant dont les lacunes sont avant tout préjudiciables aux cultivateurs. Le crédit (du latin credere, croire) repose sur la croyance, celle du prêteur à être remboursé par un créancier qui compte se tirer d'affaire en sollicitant un emprunt. Or, dans un contexte de pauvreté, la peur d'être dans l'incapacité de rembourser voire de perdre ses biens d'un côté, et la méfiance de ne pas récupérer la somme prêtée de l'autre, contraignent fortement la pratique du crédit. Moins de $12 \%$ des paysans déclarent avoir accès au crédit à travers une organisation de microcrédit. Il a été fait mention de la défiance des paysans face aux mouvements coopératifs. On retrouve ce sentiment envers les structures de microcrédit. De plus, pour épargner, encore faut-il avoir de l'argent dédié à cela. Pourtant le gouvernement soutient, du moins dans sa rhétorique, le regroupement des personnes dans des SACCOS (Saving And Credit Cooperation Structure). Ce sont des associations de microfinance basées sur un modèle participatif. En 2011, dans les trois districts de la zone d'étude, d'après les données du service régional des coopératives, seulement 31319 membres étaient enregistrés dans une SACCOS. L'adhésion demeure modique si on la rapporte à la population totale de ces trois districts qui est proche du million d'habitants. Qu'en est-il du soutien collectif, de l'entraide ? Nous disposons de peu de données dans le domaine strictement agricole. Dans le commerce ambulant rural, les colporteurs ruraux d'extraction paysanne constituent le plus souvent $(55 \%)$ eux-mêmes leur capital de départ par le salariat dans l'agricole, dans la construction, par le tout petit commerce ; un tiers a bénéficié de dons et $12 \%$ ont perçu un prêt sans intérêt issu du cercle d'affinités. En dépit des nombreux aspects troublant de l'accès au marché, la paysannerie s'y intègre en organisant sa production autour de logiques marchandes et de l'informalité entretenue par des acteurs privés du bas. Les myriades d'intermédiaires assurant la liaison entre production et commercialisation finale opèrent le plus souvent dans l'informalité, car pour l'essentiel leurs transactions ne sont pas enregistrées. En ce sens, les dynamiques marchandes du vivrier commercialisé se distinguent de l'extraversion des agricultures nationales par leurs dynamiques du bas. Par ailleurs, la sujétion totale du rural à l'urbain peut être nuancée par des rapports de complémentarité entre ville et campagne, cette dernière nourrissant les villes qui dynamisent la production rurale. Le mouvement d'intégration de la campagne et de la ville s'opère pour l'essentiel par des acteurs du bas (producteurs, 
intermédiaires, commerçants) qui ont certes des positions inégales, mais qui œuvrent pour l'intégration de la périphérie agricole aux) centre(s) urbain(s). Ils seraient les acteurs du glissement de "périphérie dominée » à "périphérie intégrée », mais qu'elle soit intégrée ou dominée, la périphérie demeure dépendante [Reynaud, 1981]. Au total, l'extraversion est certes nuancée par l'intégration du rural à l'urbain, mais le monde rural demeure toujours sous-équipé, l'accumulation (pas seulement matérielle ou financière) y est limitée et l'organisation repose sur des relations dissymétriques.

\section{Conclusion : l'échange inégal est le moteur de la dynamique du système}

L'économie montagnarde repose sur l'émission de flux de produits non transformés vers des marchés extérieurs sans valorisation locale. Les incertitudes quant à l'accès au marché, sa fragmentation, l'opacité de l'information et l'informalité des transactions indiquent de fortes dissymétries entre les acteurs et entre les territoires. Le libéralisme, la disponibilité d'un marché et des conditions environnementales favorables n'impliquent pas un enrichissement systématique de la paysannerie montagnarde qui reste cantonnée aux fonctions de production. Le processus d'accumulation reste limité dans le rural. Les producteurs ne capitalisent pas suffisamment pour s'occuper de la commercialisation dans les villes et renforcer leurs capacités de négociation et de décision au sein des chaînes marchandes. Les étapes de mise en vente sont accaparées par les acteurs privés qui se multiplient et qui sont en concurrence.

L'orientation économique dans les montagnes Uporoto illustre des mécanismes d'extraversion économique à une autre échelle : « les structures de l'économie de traite mise en place au moment de la première mondialisation, à l'époque moderne, sont restées étonnamment stables : l'Afrique exporte des produits bruts et importe des biens manufacturés » [Magrin, 2013, p. 21-22]. Certes, les éléments changent selon l'échelle, par exemple l'extraversion économique des Uporoto repose plus sur des acteurs du bas que l'extraversion nationale. Pour autant, ces relations relèvent de rapports de domination : le centre reproduit les conditions de sa centralité et l'échange inégal est le moteur de la dynamique du système. Le système spatial composé de la périphérie montagnarde et des marchés urbains, ou le «système-monde » [Durand et al., 1993; Wallerstein, 2006] donnent à voir les inégalités observées dans les relations économiques. Ces analogies entre l'économie des Uporoto et l'économie africaine soulignent aussi la dimension systémique des phénomènes locaux en interrelations avec la mondialisation, cela rappelle le principe de redondance pensé par Yves Barel [Barel, 1979], à savoir que l'on retrouve dans les parties l'expression de la totalité. En d'autres termes, des traits de l'économie rentière africaine se retrouvent dans certains traits de l'économie des montagnes sans que l'une soit réductible à l'autre. Le local est spécifique, mais il intègre ce qui se passe à d'autres échelles. D'autre part, dans le jeu des relations entre la campagne et la ville, les dissymétries de pouvoir sont en faveur de la seconde qui notamment grâce à la concentration du capital, bénéficie d'un éventail d'alternatives plus vaste que celui de la campagne. Puisque la ville et la campagne sont imbriquées, il semble donc pertinent de les associer dans les analyses.

Les perspectives sont ambivalentes. L'urbanisation est motrice du dynamisme de l'agriculture et offre de nouvelles opportunités économiques et sociales en particulier pour les jeunes, d'autant plus quand ces derniers font face à des blocages fonciers. D'un autre côté, le cadre économique dérégulé met en concurrence les territoires de production. Rien ne garantit qu'une autre région n'évince les Uporoto dans l'approvisionnement de Dar es-Salaam, puisqu'il arrive que des zones productives soient supplantées par d'autres plus compétitives, les désindustrialisations et délocalisations ici ou là, évoquant la sujétion des territoires aux logiques concurrentielles. Enfin, les intermédiaires situés à la charnière de l'urbain et du rural sont les acteurs qui profitent le plus du contrôle des flux. Cela rappelle que la mainmise sur les circulations est une rente séculaire si l'on s'en réfère à l'histoire africaine à travers laquelle les empires, royaumes et autres entités politiques les plus puissants furent ceux situés à la charnière entre le continent et le reste du monde. 


\section{Bibliographie}

BAREL [1979], LE PARADOXE ET LE SYSTEME, ESSAI SUR LE FANTASTIQUE SOCIAL, GRENOBLE, PRESSES UNIVERSITAIRES DE GRENOBLE.

BART F. [2001], « Montagnes entre marginalité et intégration », in VEYRET Y. (coord.), Les montagnes, discours et enjeux géographiques, Paris, SEDES, p. 51-69.

BAYART J.-F. [1999], «L'Afrique dans le monde: une longue histoire d'extraversion », Critique internationale, vol. 5, $\mathrm{n}^{\circ} 1$, p. 97-120.

Chaleard J.-L. [1996], Temps des villes, temps des vivres. L'essor du vivrier marchand en Côte d'Ivoire, Paris, Karthala.

Chaleard J.-L. [1999], « Les conditions de viabilité de l'agriculture paysanne », in HAUBERT M. (dir), L'avenir des paysans, les mutations des agricultures familiales dans les pays du Sud, Paris, PUF, p. 93-107.

Charlery de la Masseliere B., Nakileza B., Uginet E. [2009], « Le développement du maraîchage en Afrique de l'Est : les enjeux », Les Cahiers d'Outre-Mer, vol. 62, n 247 , p. 311-330.

Charlery de la Masseliere B., Racaud S. [2012], « De la terre-patrimoine à la terreressource : tensions entre structures foncières héritées et nouvelles perspectives des acteurs paysans en Afrique de l'Est », Bulletin de l'Association des géographes français, 2012-3, p. $412-426$.

DiJSKTRA T. [1999], « Horticultural marketing in Kenya », in VAN DER LAAN L., DiJSKTRA T. (dir.), Agricultural marketing in Tropical Africa. Contributions from the Netherlands, Leyde, African Studies Centre, no 15, p. 169-183.

Durand M.-F., LeVy J., Retaille D. [1993], Le monde, espaces et systèmes, Paris, Presses de la Fondation nationale des sciences politiques (FNSP), Dalloz.

Echaudemaison C.-D. (dir) [2013], Dictionnaire d'économie et des sciences sociales, Paris, Nathan.

Hugon P. [2009], L'économie de l'Afrique, Paris, La découverte.

Hugon P. [2012], Géopolitique de l'Afrique, Paris, SEDES.

ILIFFE J. [2009], Les Africains, histoire d'un continent, Paris, Flammarion.

Magrin G. [2013], Voyage en Afrique rentière. Une lecture géographique des trajectoires du développement, Paris, Publications de la Sorbonne.

MinVIELlE J.-P. [1999], «L'articulation des paysans au marché », in HAUBERT M. (dir.), L'avenir des paysans. Les mutations des agricultures familiales dans les pays du Sud, Paris, Puf, p. 107-123.

MORIN E. [1977], La Méthode, 1. La nature de la nature, Paris, Seuil.

PONTE S. [1998], « Fast crops, fast cash : market liberalization and rural livelihood in Songea and Morogoro Districts, Tanzania », Canadian Journal of African Studies, vol. 32, n ${ }^{\circ}$, p. 316-348.

RACAUD S. [2013], Les montagnes Uporoto entre ville et campagne. Géographie des flux et intégration territoriale en Tanzanie, thèse de géographie et aménagement, Toulouse, université Toulouse 2-le Mirail.

Reynaud A. [1981], Société, espace et justice, Paris, PUF.

SOKONI C. [2001], The influence of agricultural marketing reforms on highland farming systems in Tanzania. The case of the Uporoto Highlands, Mbeya Region, thesis of doctorate, Dar es-Salaam, university of Dar es-Salaam. 
SoKoni C. [2008], « Commercialisation of smallholder production in Tanzania : implications for sustainable resources management», The geographical journal, vol. 174, $\mathrm{n}^{\circ} 2$ : http://onlinelibrary.wiley.com/doi/10.1111/j.1475-4959.2008.00287.x/full (Page consultée le 28 septembre 2015).

United RePublic of TANZANia [2011], Mbeya regional socio economic profile, Mbeya, Mbeya regional commissioner's office.

United Republic of Tanzania [2013], 2012 Population and housing census, Dar es Salaam, national bureau of statistics, ministry of Finance, Government of Tanzania.

WALlerstein I. [2006], Comprendre le monde, Paris, La Découverte. 